\title{
p-STAT3 in luminal breast cancer: Integrated RNA-protein pooled analysis and results from the BIG 2-98 phase III trial
}

\author{
AMIR SONNENBLICK ${ }^{1}$, ROBERTO SALGADO ${ }^{2}$, SYLVAIN BROHÉE $^{2}$, TAMAR ZAHAVI ${ }^{1}$, TAMAR PERETZ ${ }^{1}$, \\ GERT VAN DEN EYNDEN ${ }^{2}$, GHIZLANE ROUAS ${ }^{2}$, ASHER SALMON $^{1}$, PRUDENCE A. FRANCIS $^{3}$, \\ ANGELO DI LEO ${ }^{4}$, JOHN P.A. CROWN ${ }^{5}$, GIUSEPPE VIALE ${ }^{6}$, LAURA DALY ${ }^{7}$, BAHAR JAVDAN $^{7}$, \\ SHO FUJISAWA ${ }^{7}$, EVANDRO DE AZAMBUJA ${ }^{2}$, AMEYE LIEVEKE ${ }^{2}$, MARTINE J. PICCART $^{2}$, \\ JACQUELINE F. BROMBERG ${ }^{7}$ and CHRISTOS SOTIRIOU ${ }^{2}$ \\ ${ }^{1}$ Sharett Institute of Oncology, Hadassah-Hebrew University Medical Center, Jerusalem 91120, Israel; \\ ${ }^{2}$ Breast Cancer Translational Research Laboratory J.-C. Heuson, Institut Jules Bordet, Université Libre de Bruxelles \\ 1000 Brussels, Belgium; ${ }^{3}$ Peter MacCallum Cancer Centre, Melbourne VIC 3000, Victoria, \\ on behalf of The Australian and New Zealand Breast Cancer Trials Group, Newcastle, NSW 2298, Australia, and \\ International Breast Cancer Study Group, 3008 Bern, Switzerland; '`Sandro Pitigliani’ Medical Oncology Department, \\ Hospital of Prato, Istituto Toscano Tumori, 50139 Firenze, Prato, Italy; ${ }^{5}$ St. Vincet's University Hospital, Elm Park, \\ on behalf of the Irish Clinical Oncology Research, Dublin 4, Ireland; ${ }^{6}$ Division of Pathology, \\ European Institute of Oncology, 20146 Milano, Italy; ${ }^{7}$ Department of Medicine, \\ Memorial Sloan Kettering Cancer Center (MSKCC), New York, NY 10065, USA
}

Received September 10, 2017; Accepted November 15, 2017

DOI: $10.3892 /$ ijo.2017.4212

\begin{abstract}
In the present study, in order to investigate the role of signal transducer and activator of transcription 3 (STAT3) in estrogen receptor (ER)-positive breast cancer prognosis, we evaluated the phosphorylated STAT3 (p-STAT3) status and investigated its effect on the outcome in a pooled analysis and in a large prospective adjuvant trial. By using the TCGA repository, we developed gene signatures that reflected the level of p-STAT3. Using pooled analysis of the expression data from luminal breast cancer patients, we assessed the effects of the p-STAT3 expression signature on prognosis. We further validated the p-STAT3 prognostic effect using immunohistochemistry (IHC) and immunofluorescence staining of p-STAT3 tissue microarrays from a large randomised prospective trial. Our analysis demonstrated that p-STAT3 expression
\end{abstract}

Correspondence to: $\mathrm{Dr}$ Amir Sonnenblick, Sharett Institute of Oncology, Hadassah-Hebrew University Medical Center, Ein-Karem, Jerusalem 91120, Israel

E-mail: amirson@hadassah.org.il

Professor Christos Sotiriou, Breast Cancer Translational Research Laboratory J.-C. Heuson, Institut Jules Bordet, Université Libre de Bruxelles, Boulevard de Waterloo, 1000 Brussels, Belgium

E-mail: christos.sotiriou@bordet.be

Key words: signal transducer and activator of transcription 3, tyrosine phosphorylation, prognostic marker, adjuvant, chemotherapy, breast cancer, estrogen receptor, luminal cancer was elevated in luminal A-type breast cancer (Kruskal-Wallis test, $\mathrm{P}<10 \mathrm{e}^{-10}$ ) and was significantly associated with a good prognosis $\left(\log -r a n k, \mathrm{P}<10 \mathrm{e}^{-10}\right)$. Notably, the p-STAT3 expression signature identified patients with a good prognosis irrespective of the luminal subtype (log-rank: luminal A, $\mathrm{P}=0.026$; luminal $\mathrm{B}, \mathrm{P}=0.006$ ). $\mathrm{p}-\mathrm{STAT} 3$ staining by $\mathrm{IHC}$ in the stroma or tumour was detected in 174 out of 610 ER-positive samples (28.5\%) from the BIG 2-98 randomised trial. With a median follow-up of 10.1 years, p-STAT3 was associated with a reduced risk of recurrence in ER-positive/HER2-negative breast cancer (Cox univariate HR, 0.66; 95\% CI, 0.44-0.98; $\mathrm{P}=0.04)$. On the whole, our data indicate that $\mathrm{p}-\mathrm{STAT} 3$ is associated with an improved outcome in ER-positive breast cancer.

\section{Introduction}

Signal transducers and activators of transcription (STATs), when phosphorylated at tyrosine elements form functional dimmers with each other, translocate to the nucleus to regulate the expression of genes by binding to specific elements within gene promoters $(1,2)$. The function of the interleukin-6 (IL-6)-STAT3 pathway in signalling and its control of the expression of different genes prompted the investigation of the pathway in cancer. Indeed, the IL-6-STAT3 pathway has been found to be critical for tumour development through different mechanisms that include direct effects on cell survival, migration and proliferation, as well as non-direct effects on the microenvironment surrounding the tumour (3-7). Although preclinical data suggest that the IL-6-STAT3 pathway is an important target for cancer therapy, to date, the practical clinical utility and efficacy is limited $(8,9)$. This 
reflects the challenges in translating preclinical evidence into relevant clinical practice and demonstrates the complexity of cancer biology.

STAT3 plays a role in the development of breast cancer, as shown by several studies $(3,4,10-13)$. However, some studies evaluating the prognostic role of phosphorylated STAT3 (p-STAT3), have yielded conflicting results (14-18). The aim of this study was to evaluate the prognostic role of p-STAT3 in luminal breast cancer patients in the adjuvant setting and in the context of a prospective trial.

\section{Materials and methods}

Computation of $p$-STAT3 reverse phase protein array (RPPA)-based signature. We analysed clinicopathological, normalised gene expression and RPPA data from the TCGA repository using its online bioinformatics tool (19). Estrogen receptor (ER)-positive breast cancers were analysed based on the RPPA proteomic levels. A total of 265 samples with available gene expression and RPPA data were considered as luminal (166 luminal $\mathrm{A}$ and 99 luminal $\mathrm{B}$ ) according to the PAM50 computed on the cBioPortal website (20). Each of the RPPA assay samples available was assigned to one of two sample groups: 'Low p-STAT3 expression' samples correspond to those samples with RPPA expression smaller than the first quartile of all expression values for this RPPA assay, while 'high p-STAT3 expression' samples correspond to those samples with a RPPA expression greater than the third quartile. To identify the genes that were differentially expressed between the low- and high-expression groups, we performed a differential gene expression analysis using a Student's t-test comparing high vs. low p-STAT3 expression tumours using a Welch t-test with robust estimators of the mean and the standard deviation.

To identify the genes that would optimise the predictive power of our signatures, we evaluated using a nested 10-fold cross validation, the maximal Benjamini-Hochberg false discovery rate and the minimal gene fold change that would optimise the ability of the differentially expressed genes to predict the high/low status of the RPPA in luminal A and B patients together and separately. While the parameters were selected in a 10-fold cross validation, the procedure itself was assessed using a nested cross validation (21). This nested procedure allowed us to remove those RPPA assays that could not deliver signatures that we could further use for prediction (ROC curves AUC $<0.6$ in luminal A, B or A and B). After this process, we were left with 69 signatures presenting a relevant AUC for proteomic status prediction. Among others, p-STAT3 achieved significant prediction ability in both luminal A and $\mathrm{B}$ cancers. The expression levels of these signatures in the gene expression datasets were computed as previously described (22).

Patients and study design. The Breast International Group (BIG) 2-98 (ClinicalTrials.gov identifier of BIG 2-98: NCT00174655), is a multicenter, prospective, open-labelled, phase III adjuvant trial that randomly assigned patients to either anthracycline-based chemotherapy or taxane combinations (23). Women received definitive surgical treatment (mastectomy or breast-conserving surgery) for invasive breast adenocarcinoma with $\geq 1$ positive axillary lymph nodes of $\geq 8$ resected nodes. All patients provided written informed consent prior to study entry. The full details and the CONSORT diagram were previously reported (24).

Central pathology review and tissue microarray (TMA) construction. A primary tumour sample (blocks or slides) was required for the central pathology review. Primary tumour samples were stored centrally at the Institut Jules Bordet (Brussels, Belgium). The central pathology review was carried out at the European Institute of Oncology (Milan, Italy). The tumour grade was centrally reviewed. Immunostaining experiments for the localization of ER and progesterone receptor $(\mathrm{PgR})$ and HER2 protein were carried out on consecutive tissue sections using an automated immunostainer (Autostainer; Dako, Glostrup, Denmark). The following primary antibodies were used: 1D5 monoclonal antibody (mAb) to ER (at 1/100 dilution), 1A6 mAb to PgR (1/800) and polyclonal antiserum (1/800) (all from Dako) to HER2 protein (23).

Only nuclear reactivity was taken into account for ER and $\mathrm{PgR}$, and the results were recorded as the percentage of immunoreactive cells over at least 2,000 neoplastic cells. Fluorescent in situ hybridization (FISH) was carried out for HER2 according to the manufacturer's instructions (Vysis; Abbott Laboratories, Abbott Park, IL, USA). Positivity thresholds were ER $\geq 1 \%$; $\operatorname{PgR} \geq 1 \%$; HER $2=3^{+}$( $>10 \%$ invasive tumour cells with intense and circumferential membrane staining) and/or FISH-positive (HER2:CEP17 ratio $\geq 2$ ).

p-STAT3 staining. The biomarker protocol for the evaluation of p-STAT3 phosphorylation in association with the clinical outcome was approved by the Institutional Review Board of Hadassah Medical Center and the BIG2-98 Study Steering Committee. From the 2,887 patients randomised in the BIG 2-98 trial, 2,173 cases had tumour blocks that were centrally evaluated. A TMA was constructed from 950 blocks.

Paraffin blocks were submitted to the coordinating center and 4 cores from each tumour were collected and placed in 2 different TMAs; each TMA contains 2 cores of the same tumour. Two laboratories performed independent staining for p-STAT3 [immunohistochemistry (IHC) and immunofluorescence (IF)]: Each laboratory received a set of available BIG2-98 specimens in TMAs. The BIG 2-98 TMA set contained 19 slides with $\sim 170$ tissue cores per slide. Two slides containing ER-negative samples were of low quality, and although stained, could not be annotated. In total, 610 and 585 ER-positive samples were interpretable for p-STAT3 by IHC and IF, respectively.

IHC was performed experimentally. The tissue microarray sections slides were deparaffinised with xylene rinses and then transferred through two changes of $100 \%$ ethanol. Endogenous peroxidase activity was blocked by a 5-min incubation in a $3 \%$ hydrogen peroxide buffer. Antigen retrieval was performed using Tris-EDTA buffer (10 mM Tris Base, $1 \mathrm{mM}$ EDTA solution). Following antigen retrieval, the slides were incubated with blocking buffers at room temperature to reduce non-specific background staining and then incubated with primary antibody at $4^{\circ} \mathrm{C}$ overnight (1:100 dilution of p-STAT3 antibody; cat. no. 9145-D3A7-XP; Cell Signaling Technology, 
Beverly, MA, USA) followed by 30 min of incubation with the secondary anti-rabbit antibody (Histofine ${ }^{\circledR}$ Simple Stain ${ }^{\mathrm{TM}}$ MAX PO (R), cat. no. 414141F; Nichirei Biosciences Inc., Tokyo, Japan). Staining was visualised using DAB.

The IHC nuclear p-STAT3 staining was determined and scored separately for each spot and specimen by two pathologists (R.S. and G.V.E.) who were blinded to the clinical pathological data and reached an agreed score for each spot. The staining was analysed according to the $\mathrm{H}$-score (range, 0-300; intensity 1-3 x percentage of positive cells $1-100$ ). For each spot, separate scores where provided for the tumour and stromal parts. For specimens that were uninterpretable, a score of 'not applicable' (N/A) was assigned. To define tumours as p-STAT3-positive, a cut-off point of $>0 \mathrm{H}$ score was selected, as well as analysing the data as a continuous variable. Specificity/sensitivity, performance and reproducibility tests of the p-STAT3 IHC were performed. Full slides from representative positive $(n=9)$ and negative $(n=8)$ TMAs were stained processed, and evaluated similarly as the experimental slides, for the indication of reproducibility and performance of the TMAs. In total, $8 / 9$ positive TMAs were found to be positive on the full slides and $8 / 8$ of the negative TMAs were found to be negative on the full slides, demonstrating $100 \%$ sensitivity and $89 \%$ specificity. As the controls for our procedure, we used HeLa cells without treatment that served as a negative control and serum-starved HeLa cells prepared with interferon- $\alpha(\mathrm{IFN}-\alpha)$ treatment that served as a positive control (all control slides were purchased from Cell Signaling Technology). The control slides were used for qualifying the procedure and not as an interpretation reference. The presence of a brown reaction product at the cell nucleus was indicative of positive reactivity.

In parallel, TMAs were stained using IF at the laboratory of J.F.B. For IF staining, the TMAs were deparaffinised and processed using the automated Ventana deparaffinization solution (Ventana Medical Systems, Inc., Tucson, AZ, USA) and $\mathrm{CC} 1$ antigen retrieval. The tissue sections were blocked for $30 \mathrm{~min}$ in $10 \%$ normal goat serum, $2 \%$ BSA in phosphate-buffered saline (PBS). The incubation with the p-STAT3 antibody was carried for $2 \mathrm{~h}$, followed by $16 \mathrm{~min}$ of biotinylated goat anti-rabbit IgG (cat. no. PK6101; Vector Laboratories, Inc., Burlingame, CA, USA) at a 1:200 dilution $(=7.5 \mu \mathrm{g} / \mathrm{ml})$. Streptavidin-HRP (Ventana Medical Systems, Inc.) was applied for 12 min followed by incubation with Tyramide-Alexa Fluor 488 (cat. no. T20922; Invitrogen, Carlsbad, CA, USA) for 16 min. p-STAT3 antibody was from Cell Signaling Technology (cat. no. 9145-D3A7-XP) a rabbit monoclonal has been purchased in large amounts $(5 \mathrm{ml})$, mixed and re-aliquoted to avoid batch-batch variation. All slides were in the same run.

The IF results were analysed using a semi-supervised freescoring tool by a digital imaging system. Specifically, each slide was scanned using Pannoramic Flash (3DHistech Ltd., Budapest, Hungary) and each tumour/stromal section was exported into a tif. image by Pannoramic Viewer (3DHistech Ltd.). Image analysis was performed using Metamorph (Molecular Devices, Sunnyvale CA, USA) in which the number of nuceli was counted using the DAPI channel, and the level of green fluorescence (p-STAT3)/nuclei was quantified.
Tissue microarray construction, the determination of the proteomic status, patient selection, assay performance and data analysis are reported according to the Recommendations for Tumour Marker Prognostic Studies (REMARK) criteria (25).

Statistical analysis. In total, 39 gene expression datasets of expression profiles from $>7,000$ tumours were retrieved from public databases or authors' websites [36 previously described (26) and another 3 sets: PNC, METABRIC and TCGA $(19,27,28)]$. To ensure the comparability of the expression values across multiple data sets, we performed a 0.95 quantile normalization. Differences in the p-STAT3 expression signatures according to subtype were examined using the Kruskal-Wallis test. Distant metastasis-free survival was the primary survival end-point, which is defined as the time elapsing between breast cancer diagnosis and the date of systemic relapse. When distant metastasis-free survival data were not reported, relapse-free survival information was used if available. Survival plots according to the p-STAT3 signatures tertiles were drawn using the Kaplan-Meier method, and the significance of the survival differences were evaluated using the log-rank P-test. The association of the signatures with good or bad prognosis were computed using uni- or multivariate Cox regression analyses. All analyses were performed using the genefu package of R (v3.2)/Bioconductor (v1.18) statistical suite.

For the BIG 2-98 outcome analysis, patients were classified according to the presence of p-STAT3. The primary outcomes were disease-free survival (DFS) and overall survival (OS). DFS was defined as the interval from the date of randomization to the date of local, regional or metastatic relapse or second primary cancer or death for any cause. OS was calculated from the date of randomization to last follow-up or death from any cause. Univariate and multivariate models were computed with the use of Cox proportional-hazards regression. The Chi-square test for categorical data and unpaired Student's t-test for continuous variables were used in order to determine an association between p-STAT3 and clinical pathological parameters, and P-values $<0.05$ were considered to indicate statistically significant differences.

\section{Results}

Association of the p-STAT3 and p-STAT3 gene expression signature with clinical parameters and outcome in patients with ER-positive breast cancer. To capture the clinical importance of p-STAT3 in ER-positive breast cancer, we analysed breast cancer samples from the TCGA repository (19). Using the PAM50 classification model, patients were assigned to the main luminal breast cancer molecular subtypes: Luminal A and luminal B. We first assessed whether p-STAT3 expression (by RPPA) was associated with a particular subtype. Our analysis revealed that luminal A-type cancers were more likely to possess p-STAT3 high levels in comparison to luminal B-type cancers (Wilcoxon $\mathrm{P}=3 \mathrm{e}^{-6}$ ) (Fig. 1A).

We then derived a gene signature whose expression levels could predict adequately the p-STAT3 RPPA levels by computing the differentially expressed genes between tumour samples with high (upper quartile) and low (lower quartile) RPPA levels of p-STAT3. To capture the clinical relevance 


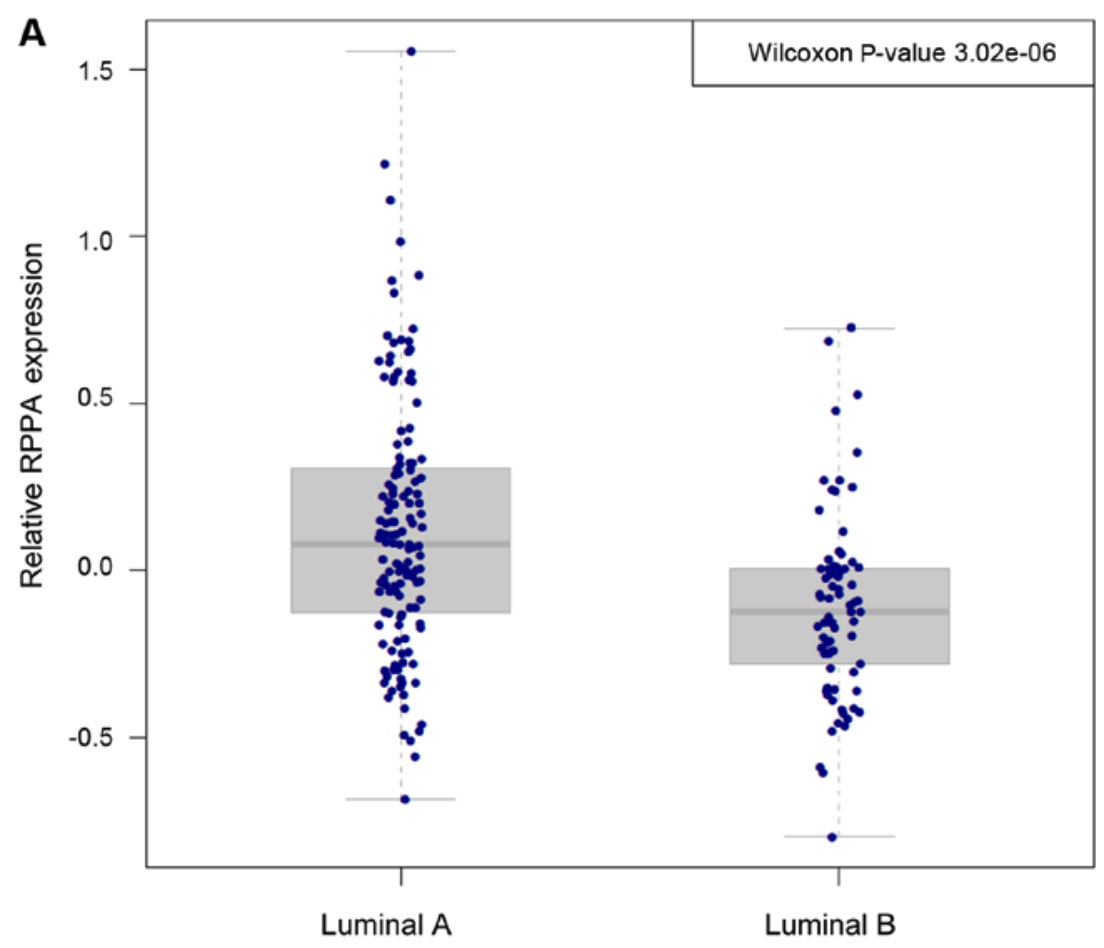

\section{B}

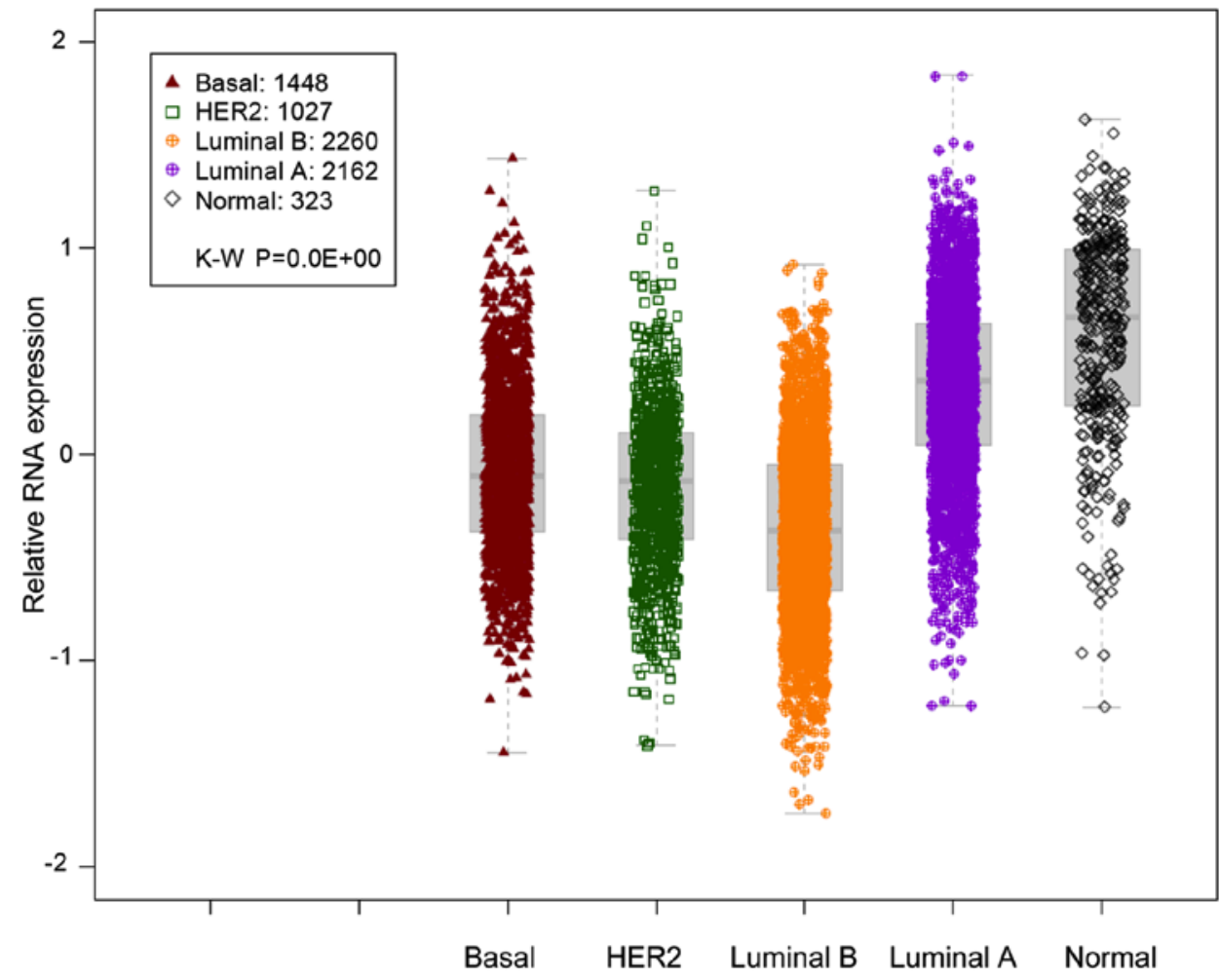

Figure 1. Analysis of phosphorylated-signal transducer and activator of transcription 3 (p-STAT3) breast cancer samples from 39 datasets. (A) Using the PAM50 classification model, patients were assigned to the main luminal breast cancer molecular subtypes: Luminal A and luminal B. Luminal A-type cancers were more likely to possess high p-STAT3 levels in comparison to luminal B-type cancers (Wilcoxon $\mathrm{P}=3 \mathrm{e}^{-6}$ ). (B) p-STAT3 gene expression signatures in publically available microarray datasets according to the PAM50 breast cancer subtype. Kruskal-Wallis $\mathrm{P}$-value is shown $\left(\mathrm{P}<0.01^{-10}\right)$.

of the p-STAT3 expression signature in ER-positive breast cancer, we combined 39 publically available microarray datasets comprising over 7,000 breast cancer patients to build a pooled set of gene-expression profiles with available outcome data. Using the PAM50 classification model, patients were assigned to the main breast cancer molecular subtypes, namely luminal A, luminal B, HER2-enriched, basal-like and normal-like breast cancers. We first assessed whether the p-STAT3 expression signatures were associated with any particular luminal subtype. As expected, in the pooled set 

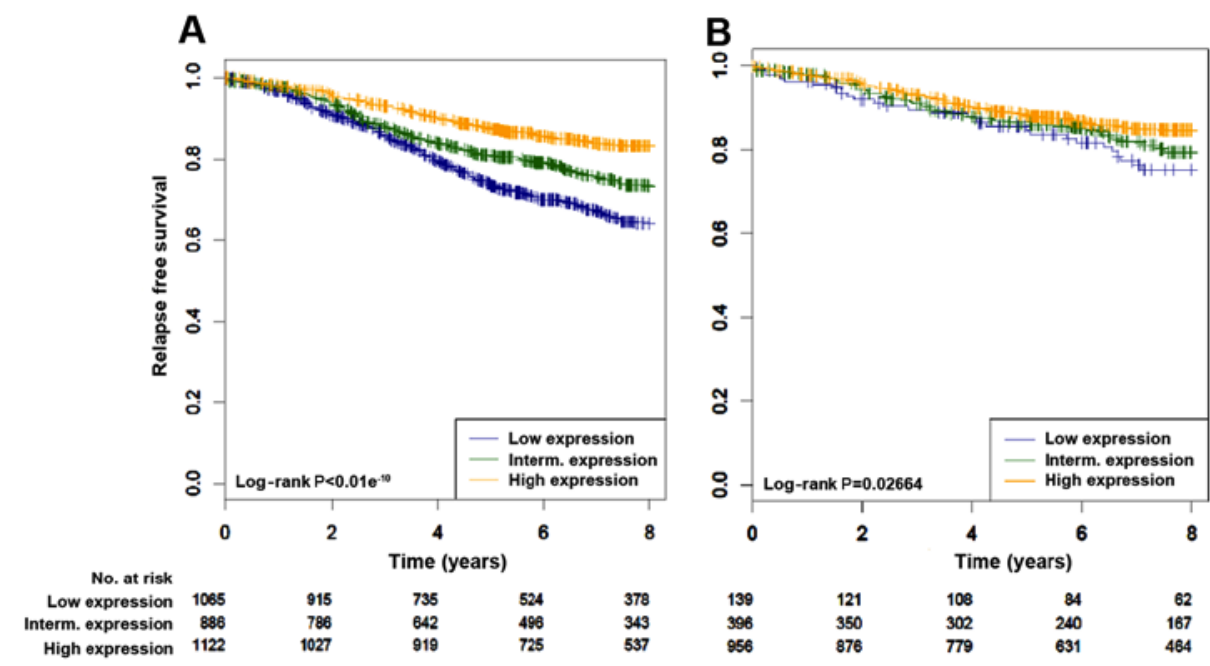

D

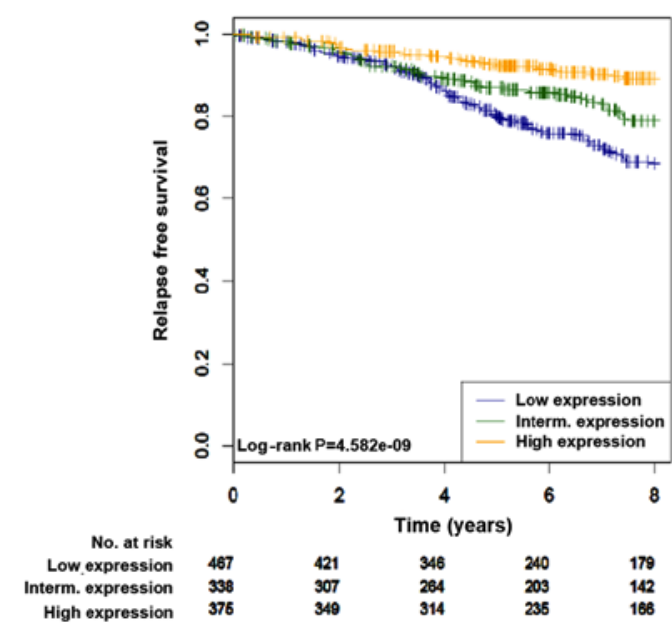

E

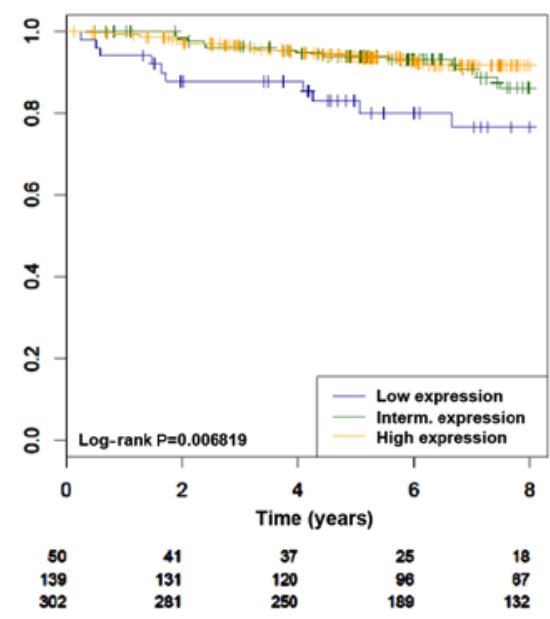

C

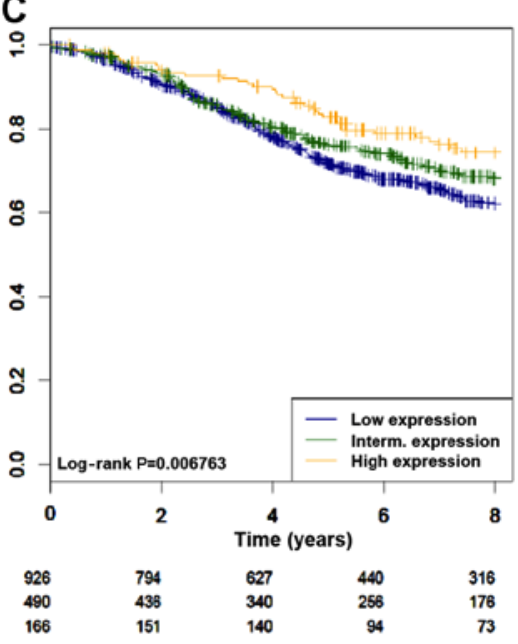

$\mathbf{F}$

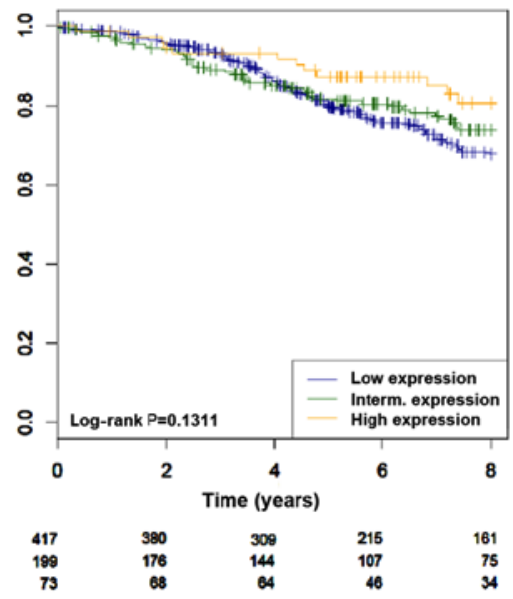

Figure 2. High phosphorylated-signal transducer and activator of transcription 3 (p-STAT3) gene signature expression is associated with a good prognosis in the luminal subtype. (A-F) We assessed the prognostic value of tertiles of the p-STAT3 gene expression signature in: (A) All luminal patients treated or not treated $(n=3,073)$, (B) luminal A $(n=1,491)$, (C) luminal B $(n=1,582)$, (D) all luminal treated with only hormonal therapy $(n=1,180)(E)$ luminal A treated with only hormonal therapy $(\mathrm{n}=491)(\mathrm{F})$ luminal B treated with only hormonal therapy $(\mathrm{n}=689)$. The significance (P-values) of differences in survival between patients groups defined by tertiles of p-STAT3 signature expression was estimated by the log-rank test.

analysis, the p-STAT3 expression signature was significantly associated will luminal A-type cancers $\left(\mathrm{P}<0.01^{-10}\right)$ (Fig. 1B). We then assessed whether p-STAT3 correlated with survival in patients with ER-positive breast cancer in cohorts where relapse data was available. As shown in Fig. 2 and Table I, the p-STAT3 expression signature was significantly associated with a good prognosis in all patients with luminal tumours (log-rank, $\mathrm{P}<0.01^{-10}$ ) (Fig. 2A). Similar results were found with patients treated with hormonal therapy only (2D log-rank, $\left.\mathrm{P}=4.58 \mathrm{e}^{-9}\right)$. Of interest, the $\mathrm{p}-\mathrm{STAT} 3$ expression signature was able to identify patients with a good prognosis irrespective of the luminal subtypes (luminal $\mathrm{A}, \log$-rank, $\mathrm{P}=0.027$; luminal $\mathrm{B}, \log$-rank, $\mathrm{P}=0.006$ ) (Fig. $2 \mathrm{~B}$ and $\mathrm{C}$ ). Cox multivariate analysis confirmed the independent prognostic value of p-STAT3 (Table I).

Association of p-STAT3 with clinicopathological characteristics and outcome in the BIG 2-98 randomised trial. We then confirmed our observations at the proteomic level in the context of prospective data from the BIG 2-98 repository. There were 610 and 585 ER-positive tumour TMAs available for the evaluation of p-STAT3 by IHC and IF, respectively. Evaluation was performed for the tumoral and stromal component separately (Fig. 3). Any level of p-STAT3 staining by IHC in the tumour or stroma was detected in 174 out of the 610 samples (28.5\%). p-STAT3 (in the tumour or stroma) was associated with a smaller tumour size when analysed by IHC (Table II) or IF (data not shown). p-STAT3 expression between the tumour and stroma strongly correlated using IHC ( $\mathrm{R}=0.67$; 95\% CI, 0.63-0.71) or IF ( $\mathrm{R}=0.86$; 95\% CI, 0.84-0.88) as evaluated by Spearman's correlation. For the IF analysis, two patterns of staining were found (patchy and diffuse) that were not associated with different clinical or pathological parameters (data not shown).

For the prognostic evaluations, all treatment arms were pooled. We examined p-STAT3 in the tumour or stroma using IHC and their association with DFS and OS end points. As summarised in Table III, there was no significant prognostic 
Table I. Cox univariate and multivariate analysis of relapse data from the pooled analysis according to the p-STAT3 gene expression signature status.

\begin{tabular}{llcccc}
\hline Subtype & \multicolumn{1}{c}{ Treatment } & $\begin{array}{c}\text { Hazard Cox } \\
\text { univariate }\end{array}$ & P-value & $\begin{array}{c}\text { Hazard Cox } \\
\text { multivariate }\end{array}$ & P-value \\
\hline All luminal & Treated and not treated & 0.52 & $<0.01^{-10}$ & 0.58 & $1.27 \mathrm{E}^{-09}$ \\
All luminal & Hormonotherapy treatment only & 0.43 & $3.37 \mathrm{E}^{-10}$ & 0.55 & $3.48 \mathrm{E}^{-05}$ \\
Luminal A & Treated and not treated & 0.64 & 0.004 & 0.67 & 0.059 \\
Luminal A & Hormonotherapy treatment only & 0.46 & 0.02 & 0.64 & 0.23 \\
Luminal B & Treated and not treated & 0.72 & 0.002 & 0.78 & 0.045 \\
Luminal B & Hormonotherapy treatment only & 0.64 & 0.018 & 0.76 & 0.16 \\
\hline
\end{tabular}

p-STAT3, phosphorylated-signal transducer and activator of transcription 3.

Table II. Association of p-STAT3 (by immunohistochemistry) expression with clinicopathological parameters in ER-positive breast cancer.

\begin{tabular}{|c|c|c|c|}
\hline Characteristics & $\begin{array}{l}\text { p-STAT3-negative } \\
\text { in both tumour and stroma } \\
\qquad(\mathrm{n}=436)\end{array}$ & $\begin{array}{l}\text { p-STAT3-positive } \\
\text { in tumour or stroma } \\
\qquad(\mathrm{n}=174)\end{array}$ & P-value \\
\hline \multicolumn{4}{|l|}{ Age at randomization, years } \\
\hline Mean \pm SD & $48.6 \pm 9.2$ & $48.4 \pm 8.5$ & \multirow[t]{2}{*}{0.80} \\
\hline Median (range) & $49(20-69)$ & $48(27-69)$ & \\
\hline \multicolumn{4}{|l|}{ No. of involved nodes, no (\%) } \\
\hline $1-3$ & $229(52.5)$ & $95(54.6)$ & \multirow[t]{3}{*}{0.70} \\
\hline $4-10$ & $153(35.1)$ & $58(33.3)$ & \\
\hline$>10$ & $54(12.4)$ & $21(12.1)$ & \\
\hline \multicolumn{4}{|l|}{ Tumor size, no (\%) } \\
\hline$\leq 2 \mathrm{~cm}$ & $133(30.8)$ & $72(41.6)$ & \multirow[t]{3}{*}{0.01} \\
\hline$>2 \mathrm{~cm}$ & $299(69.2)$ & $101(58.4)$ & \\
\hline pTx & 4 & 1 & \\
\hline \multicolumn{4}{|l|}{ Tumor grade, no (\%) } \\
\hline G1-G2 & $234(55.9)$ & $103(61.7)$ & \multirow[t]{3}{*}{0.20} \\
\hline G3 & $185(44.2)$ & $64(38.3)$ & \\
\hline Gx & 17 & 7 & \\
\hline \multicolumn{4}{|l|}{ PR, no (\%) } \\
\hline $\mathrm{PR}^{-}$ & $41(9.6)$ & $13(7.6)$ & \multirow[t]{3}{*}{0.44} \\
\hline $\mathrm{PR}^{+}$ & $388(90.4)$ & $158(92.4)$ & \\
\hline Missing info & 7 & 3 & \\
\hline \multicolumn{4}{|l|}{ HER 2, no (\%) } \\
\hline HER2- & $364(84.5)$ & $152(88.4)$ & \multirow[t]{3}{*}{0.21} \\
\hline $\mathrm{HER}^{+}{ }^{+}$ & $67(15.6)$ & $20(11.6)$ & \\
\hline Missing info & 5 & 2 & \\
\hline \multicolumn{4}{|l|}{ Taxane, no (\%) } \\
\hline No & $131(30.1)$ & $59(33.9)$ & \multirow[t]{2}{*}{0.35} \\
\hline Yes & $305(70.0)$ & $115(66.1)$ & \\
\hline \multicolumn{4}{|l|}{ Seq/combined, no (\%) } \\
\hline Sequential & $213(48.9)$ & $104(59.8)$ & \multirow[t]{4}{*}{0.01} \\
\hline Combined & $223(51.2)$ & $70(40.2)$ & \\
\hline \multirow[t]{2}{*}{ Median follow-up, years $(95 \% \mathrm{CI})$} & 10.45 & & \\
\hline & $(10.15-10.61) \quad(10.11-10.70)$ & 0.51 & \\
\hline No. of deaths & 98 & 34 & \\
\hline No. of events (BCR, SPM, death) & 159 & 51 & \\
\hline
\end{tabular}

BCR, breast cancer recurrence; SPM, second primary malignancies; p-STAT3, phosphorylated-signal transducer and activator of transcription 3; ER, estrogen receptor. 
Table III. Prognostic value of p-STAT3 (immunohistochemistry) using univariate analysis.

\begin{tabular}{|c|c|c|c|c|}
\hline & \multicolumn{2}{|c|}{ DFS } & \multicolumn{2}{|c|}{ OS } \\
\hline & $\operatorname{HR}(95 \% \mathrm{CI})$ & P-value & $\mathrm{HR}(95 \% \mathrm{CI})$ & P-value \\
\hline \multicolumn{5}{|l|}{ All } \\
\hline Tumour p-STAT3 ordinal ${ }^{\mathrm{a}}$ & $0.84(0.68-1.04)$ & 0.10 & $0.83(0.63-1.08)$ & 0.16 \\
\hline Tumour p-STAT3 binary (positive vs. negative) & $0.72(0.51-1.04)$ & 0.08 & $0.75(0.48-1.18)$ & 0.21 \\
\hline Stroma p-STAT3 ordinal $^{\mathrm{a}}$ & $0.85(0.68-1.05)$ & 0.13 & $0.88(0.67-1.15)$ & 0.34 \\
\hline Stroma p-STAT3 binary (positive vs. negative) & $0.78(0.55-1.08)$ & 0.14 & $0.79(0.52-1.21)$ & 0.28 \\
\hline \multicolumn{5}{|l|}{$\mathrm{ER}^{+} / \mathrm{HER} 2^{-}$} \\
\hline Tumour p-STAT3 ordinal ${ }^{\mathrm{a}}$ & $0.80(0.63-1.01)$ & 0.06 & $0.83(0.62-1.11)$ & 0.22 \\
\hline Tumour p-STAT3 binary (positive vs. negative) & $0.66(0.44-0.98)$ & 0.04 & $0.70(0.43-1.15)$ & 0.16 \\
\hline Stroma p-STAT3 ordinal $^{\mathrm{a}}$ & $0.86(0.67-1.09)$ & 0.20 & $0.92(0.68-1.22)$ & 0.55 \\
\hline Stroma p-STAT3 binary (positive vs. negative) & $0.75(0.52-1.09)$ & 0.13 & $0.82(0.52-1.30)$ & 0.39 \\
\hline \multicolumn{5}{|l|}{$\mathrm{ER}^{+} / \mathrm{HER} 2^{+}$} \\
\hline Tumor p-STAT3 ordinal ${ }^{\mathrm{a}}$ & $1.09(0.66-1.80)$ & 0.73 & $0.86(0.40-1.85)$ & 0.71 \\
\hline Tumor p-STAT3 binary (positive vs. negative) & $1.33(0.56-3.21)$ & 0.52 & $1.34(0.45-3.93)$ & 0.60 \\
\hline Stroma p-STAT3 ordinal ${ }^{a}$ & $0.86(0.49-1.52)$ & 0.61 & $0.78(0.36-1.67)$ & 0.52 \\
\hline Stroma p-STAT3 binary (positive vs. negative) & $0.93(0.41-2.12)$ & 0.86 & $0.79(0.27-2.34)$ & 0.67 \\
\hline
\end{tabular}

${ }^{a}$ Ordinal indicates 4 categories for immunohistochemistry, i.e., 0, 0.1-10, 10.1-100,>100; bold font indicates statistical significance. p-STAT3, phosphorylated-signal transducer and activator of transcription 3; ER, estrogen receptor; DFS, disease-free survival; OS, overall survival.
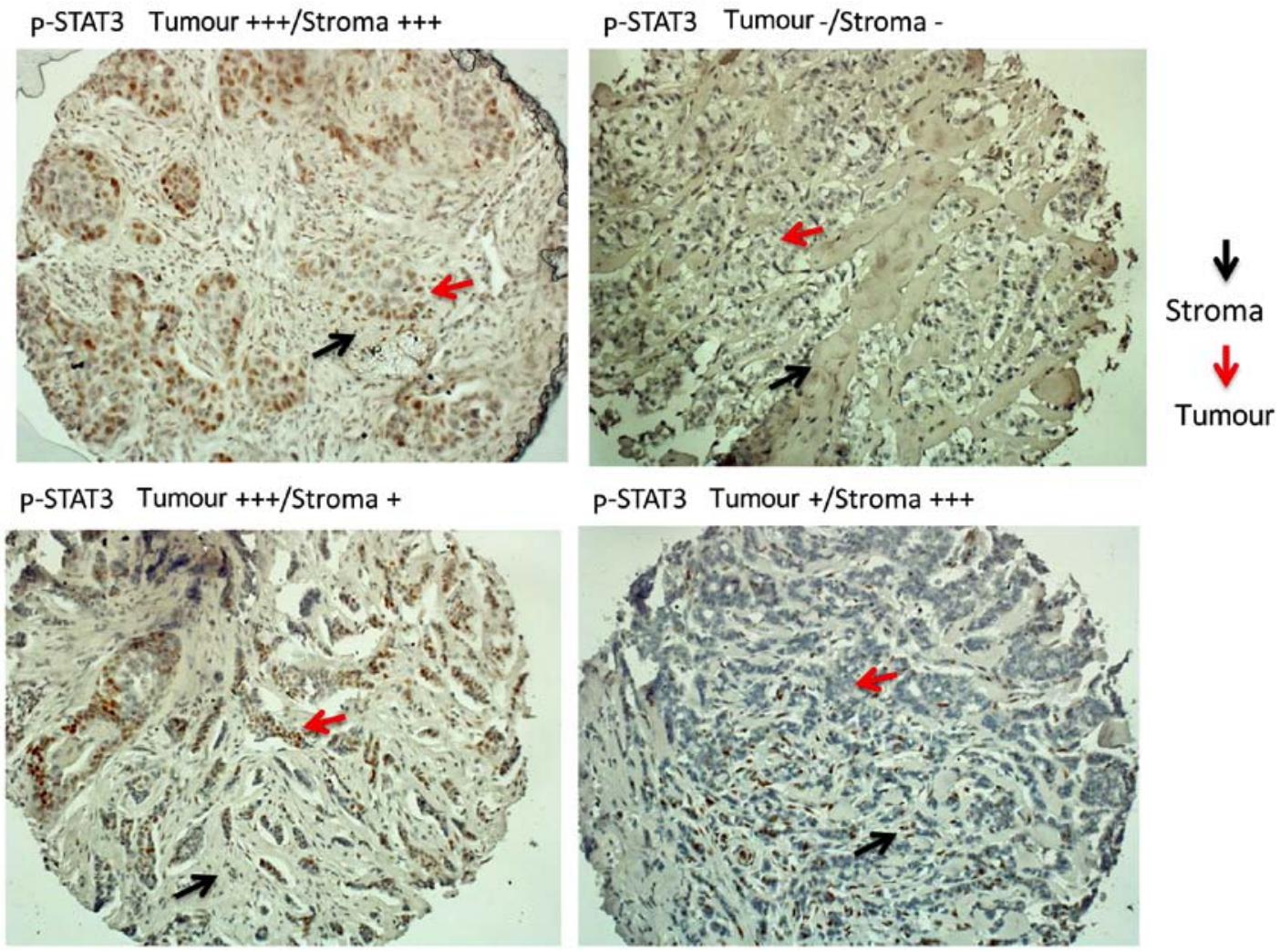

P-STAT3 Tumour + /Stroma +++

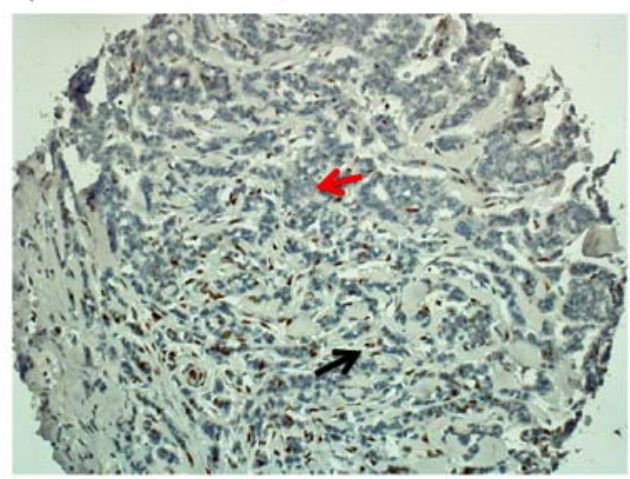

Figure 3. Tissue microarrays (TMAs) were stained for phosphorylated-signal transducer and activator of transcription 3 (p-STAT3) by immunohistochemistry. The evaluation was performed for the tumoral and stromal component separately.

effect in the global population (all ER-positive), although a trend for an improved DFS was observed (HR, 0.72; 95\% CI, 0.51-1.04; P=0.08). For the ER-positive/HER2 negative group, p-STAT3 was found to be prognostic using univariate (positive vs. negative) analysis for DFS (Cox univariate HR, 0.66; 95\% CI, 0.44-0.98; P=0.04), but not OS (Cox univariate $\mathrm{HR}, 0.70 ; 95 \% \mathrm{CI}, 0.43-1.15 ; \mathrm{P}=0.16$ ). Multivariate analysis, adjusting for the grade and number of positive lymph 
nodes, did not reach significance (Cox multivariate, HR, 0.76; 95\% CI, 0.51-1.15; $\mathrm{P}=0.19$ ). The analysis of ER-positive/HER2 negative samples from IF staining with diffuse distribution demonstrated the same trend of improved outcome in p-STAT3positive tumours (DFS: HR, 0.59; 95\% CI, 0.35-1.01, $\mathrm{P}=0.056$; OS: HR, 0.53; 95\% CI, 0.26-1.05, $\mathrm{P}=0.068$ ) (data not shown).

\section{Discussion}

In the present study, we sought to better understand the associations between p-STAT3 the luminal phenotype and outcome of breast cancer, using an approach that integrated RPPA and microarrays gene expression data. Specifically, we were interested in identifying a p-STAT3 expression signature in luminal breast cancers which could be used for prognostic purposes. Although significant data exist on p-STAT3 target genes/signatures in triple-negative breast cancer models and datasets, relatively few have been identified for luminal breast cancers (29). Notably, Walker et al determined that ER-positive tumours co-express both p-STAT5 and p-STAT3 and identified a STAT3/STAT5 expression signature (albeit using a small number of samples) distinct from STAT3 which correlated with an improved outcome (18). In this study, we did not analyse p-STAT5 levels, as they are not represented in the TCGA RPPA data set. In addition to the p-STAT3 levels, total STAT3 is considered a 'surrogate' for p-STAT3, as STAT3 is positively regulated by p-STAT3. Unfortunately, we could not perform this analysis, as the total STAT3 protein levels were similarly not available from the TCGA RPPA dataset.

We also validated the observation that p-STAT3 is associated with a better outcome in ER-positive patients randomised in the BIG 2-98 trial. We found that tumours highly expressing p-STAT3 were associated with a smaller tumour size, luminal A phenotype and a good clinical outcome in comparison to those expressing low levels of p-STAT3. In a recent study, it was suggested that patients in the luminal A population were much more likely to possess a p-STAT3 high phenotype, but this was not associated with specific genes that were differentially expressed between the p-STAT3 high vs. low groups (29). Therefore, it was hypothesised that although STAT3 is activated variably between tumour subtypes, the overall mechanisms through which STAT3 affects gene expression are multifactorial and may not entirely correlate with STAT3 phosphorylation.

In a recent study, hormonal treatment did not improve outcome in patients with increased levels of protein inhibitor of activated STAT3 (PIAS3). It was suggested that an increased PIAS3 expression attenuates the effectiveness of tamoxifen (30). This is in support of our observation that STAT3 activation was associated with an improved outcome in ER-positive tumours.

Although our data strongly suggest that a high p-STAT3 and a p-STAT3 expression signature correlate with an improved outcome, these observations do not allow us to propose that targeting this molecule or pathway would have deleterious effects. For example, activating mutations in the PI3K pathway found predominantly in luminal breast cancers are associated with an improved clinical outcome (31). Despite this correlation, targeting this pathway with small molecule inhibitors in conjunction with anti-estrogens has led to improved outcomes in patients with metastatic disease (32).
As regards the IL-6/STAT3 signalling pathway, it was recently shown in pre-clinical models of luminal breast cancer that reversing its activity with an IL-6R blocking antibody (tocilizumab) restored a dependence on anti-estrogens through increased ER expression and elimination of CD133 stem cells (33). The potential benefits of targeting this pathway in endocrine-therapy resistant metastatic disease are presently being tested. Specifically, a clinical trial combining the JAK1/2 inhibitor ruxolitinib with endocrine therapy is ongoing (NCT01594216). Notably, the selection of inhibitor used could affect the outcome. Specifically, a JAK1/2 inhibitor can also target STAT5 and STAT1 which could influence response and prevent one from concluding that the outcome is due to targeting only STAT3.

In conclusion, this study provides evidence that the p-STAT3 status is a marker of favorable outcome in ER-positive breast cancer.

\section{Acknowledgements}

This study was funded by a Clinical Research Career Development Award from the Israel Cancer Research Fund grants (16-116-CRCDA) and from the Israeli Cancer Research Association (2017-0140).

\section{References}

1. Darnell JE Jr, Kerr IM and Stark GR: Jak-STAT pathways and transcriptional activation in response to IFNs and other extracellular signaling proteins. Science 264: 1415-1421, 1994.

2. Zhong Z, Wen Z and Darnell JE Jr: Stat3: A STAT family member activated by tyrosine phosphorylation in response to epidermal growth factor and interleukin-6. Science 264: 95-98, 1994.

3. Garcia R, Bowman TL, Niu G, Yu H, Minton S, Muro-Cacho CA, Cox CE, Falcone R, Fairclough R, Parsons S, et al: Constitutive activation of Stat 3 by the Src and JAK tyrosine kinases participates in growth regulation of human breast carcinoma cells Oncogene 20: 2499-2513, 2001.

4. Niu G, Wright KL, Huang M, Song L, Haura E, Turkson J, Zhang S, Wang T, Sinibaldi D, Coppola D, et al: Constitutive Stat3 activity up-regulates VEGF expression and tumor angiogenesis. Oncogene 21: 2000-2008, 2002.

5. Yu H, Lee H, Herrmann A, Buettner R and Jove R: Revisiting STAT3 signalling in cancer: New and unexpected biological functions. Nat Rev Cancer 14: 736-746, 2014

6. Knüpfer H and Preiss R: Significance of interleukin-6 (IL-6) in breast cancer (Review). Breast Cancer Res Treat 102: 129-135, 2007.

7. Yu H, Pardoll D and Jove R: STATs in cancer inflammation and immunity: A leading role for STAT3. Nat Rev Cancer 9: 798-809, 2009.

8. Yue P and Turkson J: Targeting STAT3 in cancer: How successful are we? Expert Opin Investig Drugs 18: 45-56, 2009.

9. Sansone $\mathrm{P}$ and Bromberg J: Targeting the interleukin-6/Jak/stat pathway in human malignancies. J Clin Oncol 30: 1005-1014, 2012.

10. Chang Q, Bournazou E, Sansone P, Berishaj M, Gao SP, Daly L, Wels J, Theilen T, Granitto S, Zhang X, et al: The IL-6/JAK/Stat3 feed-forward loop drives tumorigenesis and metastasis. Neoplasia 15: 848-862, 2013

11. Azare J, Doane A, Leslie K, Chang Q, Berishaj M, Nnoli J, Mark K, Al-Ahmadie H, Gerald W, Hassimi M, et al: Stat3 mediates expression of autotaxin in breast cancer. PLoS One 6: e27851, 2011.

12. Gritsko T, Williams A, Turkson J, Kaneko S, Bowman T, Huang M, Nam S, Eweis I, Diaz N, Sullivan D, et al: Persistent activation of stat 3 signaling induces survivin gene expression and confers resistance to apoptosis in human breast cancer cells. Clin Cancer Res 12: 11-19, 2006.

13. Burke WM, Jin X, Lin HJ, Huang M, Liu R, Reynolds RK and Lin J: Inhibition of constitutively active Stat 3 suppresses growth of human ovarian and breast cancer cells. Oncogene 20: 7925-7934, 2001. 
14. Sonnenblick A, Uziely B, Nechushtan H, Kadouri L, Galun E, Axelrod JH, Katz D, Daum H, Hamburger T, Maly B, et al: Tumor STAT3 tyrosine phosphorylation status, as a predictor of benefit from adjuvant chemotherapy for breast cancer. Breast Cancer Res Treat 138: 407-413, 2013.

15. Sonnenblick A, Shriki A, Galun E, Axelrod JH, Daum H, Rottenberg Y, Hamburger T, Mali B and Peretz T: Tissue microarray-based study of patients with lymph node-positive breast cancer shows tyrosine phosphorylation of signal transducer and activator of transcription 3 (tyrosine705-STAT3) is a marker of good prognosis. Clin Transl Oncol 14: 232-236, 2012.

16. Walker SR, Nelson EA, Yeh JE, Pinello L, Yuan GC and Frank DA: STAT5 outcompetes STAT3 to regulate the expression of the oncogenic transcriptional modulator BCL6. Mol Cell Biol 33: 2879-2890, 2013.

17. Dolled-Filhart M, Camp RL, Kowalski DP, Smith BL and Rimm DL: Tissue microarray analysis of signal transducers and activators of transcription 3 (Stat3) and phospho-Stat3 (Tyr705) in node-negative breast cancer shows nuclear localization is associated with a better prognosis. Clin Cancer Res 9: 594-600, 2003

18. Walker SR, Xiang M and Frank DA: Distinct roles of STAT3 and STAT5 in the pathogenesis and targeted therapy of breast cancer. Mol Cell Endocrinol 382: 616-621, 2014.

19. Cancer Genome Atlas Network: Comprehensive molecular portraits of human breast tumours. Nature 490: 61-70, 2012.

20. Gao J, Aksoy BA, Dogrusoz U, Dresdner G, Gross B, Sumer SO, Sun Y, Jacobsen A, Sinha R, Larsson E, et al: Integrative analysis of complex cancer genomics and clinical profiles using the cBioPortal. Sci Signal 6: pl1, 2013

21. Varma S and Simon R: Bias in error estimation when using crossvalidation for model selection. BMC Bioinformatics 7: 91, 2006.

22. Sonnenblick A, Brohée S, Fumagalli D, Rothé F, Vincent D Ignatiadis M, Desmedt C, Salgado R, Sirtaine N, Loi S, et al: Integrative proteomic and gene expression analysis identify potential biomarkers for adjuvant trastuzumab resistance: Analysis from the Fin-her phase III randomized trial. Oncotarget 6: 30306-30316, 2015.

23. Sonnenblick A, Francis PA, Azim HA Jr, de Azambuja E, Nordenskjöld B, Gutiérez J, Quinaux E, Mastropasqua MG, Ameye L, Anderson M, et al: Final 10-year results of the Breast International Group 2-98 phase III trial and the role of Ki67 in predicting benefit of adjuvant docetaxel in patients with oestrogen receptor positive breast cancer. Eur J Cancer 51: 1481-1489, 2015.

24. Francis P, Crown J, Di Leo A, Buyse M, Balil A, Andersson M, Nordenskjöld B, Lang I, Jakesz R, Vorobiof D, et al; BIG 02-98 Collaborative Group: Adjuvant chemotherapy with sequential or concurrent anthracycline and docetaxel: Breast International Group 02-98 randomized trial. J Natl Cancer Inst 100: 121-133, 2008.
25. McShane LM, Altman DG, Sauerbrei W, Taube SE, Gion M and Clark GM; Statistics Subcommittee of NCI-EORTC Working Group on Cancer Diagnostics: REporting recommendations for tumor MARKer prognostic studies (REMARK). Breast Cancer Res Treat 100: 229-235, 2006.

26. Haibe-Kains B, Desmedt C, Loi S, Culhane AC, Bontempi G, Quackenbush J and Sotiriou C: A three-gene model to robustly identify breast cancer molecular subtypes. J Natl Cancer Inst 104: 311-325, 2012.

27. Curtis C, Shah SP, Chin SF, Turashvili G, Rueda OM, Dunning MJ, Speed D, Lynch AG, Samarajiwa S, Yuan Y, et al; METABRIC Group: The genomic and transcriptomic architecture of 2,000 breast tumours reveals novel subgroups. Nature 486: 346-352, 2012.

28. Dedeurwaerder S, Desmedt C, Calonne E, Singhal SK, Haibe-Kains B, Defrance M, Michiels S, Volkmar M, Deplus R, Luciani J, et al: DNA methylation profiling reveals a predominant immune component in breast cancers. EMBO Mol Med 3: 726-741, 2011.

29. Tell RW and Horvath CM: Bioinformatic analysis reveals a pattern of STAT3-associated gene expression specific to basal-like breast cancers in human tumors. Proc Natl Acad Sci USA 111: 12787-12792, 2014

30. Yang SF, Hou MF, Chen FM, Ou-Yang F, Wu YC, Chai CY and Yeh YT: Prognostic value of protein inhibitor of activated STAT3 in breast cancer patients receiving hormone therapy. BMC Cancer 16: 20, 2016.

31. Loi S, Haibe-Kains B, Majjaj S, Lallemand F, Durbecq V, Larsimont D, Gonzalez-Angulo AM, Pusztai L, Symmans WF, Bardelli A, et al: PIK3CA mutations associated with gene signature of low mTORC1 signaling and better outcomes in estrogen receptor-positive breast cancer. Proc Natl Acad Sci USA 107: 10208-10213, 2010

32. Baselga J, Campone M, Piccart M, Burris HA III, Rugo HS, Sahmoud T, Noguchi S, Gnant M, Pritchard KI, Lebrun F, et al: Everolimus in postmenopausal hormone-receptor-positive advanced breast cancer. N Engl J Med 366: 520-529, 2012.

33. Sansone P, Ceccarelli C, Berishaj M, Chang Q, Rajasekhar VK, Perna F, Bowman RL, Vidone M, Daly L, Nnoli J, et al: Self-renewal of CD133(hi) cells by IL6/Notch3 signalling regulates endocrine resistance in metastatic breast cancer. Nat Commun 7: 10442, 2016. 